Revue québécoise de linguistique

\title{
Sur l'unité du mot et la syntaxe comparée des langues romanes : le morphème invest- en italien et en français
}

\section{Annibale Elia}

Volume 13, numéro 2, 1984

Grammaire et lexique

URI : https://id.erudit.org/iderudit/602518ar

DOI : https://doi.org/10.7202/602518ar

Aller au sommaire du numéro

Éditeur(s)

Université du Québec à Montréal

ISSN

0710-0167 (imprimé)

1705-4591 (numérique)

Découvrir la revue

\section{Citer cet article}

Elia, A. (1984). Sur l'unité du mot et la syntaxe comparée des langues romanes : le morphème invest- en italien et en français. Revue québécoise de linguistique, 13(2), 193-216. https://doi.org/10.7202/602518ar 


\title{
SUR L'UNITÉ DU MOT ET LA SYNTAXE COMPARÉE DES LANGUES ROMANES: LE MORPHÈME INVEST- EN ITALIEN ET EN FRANÇAIS
}

\author{
Annibale Elia*
}

\section{Introduction}

Un des problèmes de la syntaxe formelle est de rendre compte des structures de phrase ainsi que des aspects distributionnels, c'est-àdire de la cooccurrence des "mots" ou "morphèmes lexicaux" entre eux.

La complexité de ces phénomènes est telle que, d'une part, le syntacticien est amené à gênéraliser et à rendre abstraites les caractéristiques structurales, et d'autre part, le lexicologue (ou Ie lexicographe) est amenè à multtplier les acceptions, les emplois des différents mots ou morphèmes et donc à isoler (atomiser) tout ce que le grammairien souhaite uni et compact.

* Ce travail est en partie financé par 1'ERA 247 du C.N.R.S. et par le fonds du $60 \%$ pour la recherche du M.P.I. Italie auprès de $1^{\prime} \mathrm{U}-$ niversité de Salerno. I1 s'inscrit dans les recherches du Lessico-Grammatica della Lingua Italiana (LGLI) auprès du Centre d'informatique linguistique CILFS de $1^{\prime}$ Institut de Linguistique de l'Université de Salerno. Je remercie Maurizio Martinel11 et Emilio $D^{\prime}$ Agostino pour les suggestions pertinentes qu'ils m'ont faites et Mireille Piot pour son aide éditoriale. 
Un exemple particulièrement significatif est celui des verbes et des formes lexicales qui leur sont morphologiquement liées (par exemple, PASSEggiare - PASSEggio - PASSEggiata - PASSEggtatore - PASSEgGIATRICE). En général, le lexicographe indique les différents emplois d'un verbe ou d'un nom en mettant en évidence (par exemple avec des chiffres ou des lettres) les différentes acceptions, selon le sens. Souvent, des phrases servent d'exemple de I'emploi ou de l'acception, mais leur choix ne suit pas, en général, de méthodologie explicite: il dépend du bon sens et de la culture du lexicographe et, le plus souvent, des attestations écrites.

La construction d'un lexique-grammaire ${ }^{1}$ permet de tenir compte des restrictions de sélection et de sous-catégorisation, ainsi que des relations de morphologie dérivationnelle, sans s'éloigner du cadre de la syntaxe formelle, c'est-à-dire en limitant à la seule expérience du jugement d'acceptabilité, le recours à des intuitions de nature sémantique. Dans cette étude, nous montrerons comment un lexique-grammaire peut rendre compte, d'une façon satisfaisante, des diversités lexicales et syntaxiques du verbe investire et des noms qui lui sont morphologiquement liés. Nous donnerons aussi une esquisse de comparaison avec les verbes et les noms du français construits à partir du même morphème INVEST-.

Nous nous référerons à la notation et à la méthodologie du L.A.D. L., surtout pour ce qui concerne les notions de verbe support et de est faite dans EMDA (1981); pour le français, voir Gross (1975). 
verbe opérateur ${ }^{2}$.

2. Le verbe investire

Le verbe investire est en relation morphologique avec les noms investitura, investimento, investitore et investito (ce dernier, dêrivé du participe passê). Nous allons en examiner les différents emplois et en illustrer les propriétés structurales et lexicales.

INVESTIRE 1

(1) $\quad \mathrm{N}_{0} \vee \mathrm{N}_{1} \mathrm{di} \mathrm{N}_{2}=$ :

(1) a. La régina ha investito $i$ Beatles del titolo di baronetti

(1) b. Il governo ha investito una comissione parlamentare del compito di indagare sul caso Moro

INVESTIRE 2

(2) $\quad \mathrm{N}_{0} \vee \mathrm{N}_{1}$ in $\mathrm{N}_{2}=$ :

(2) a. Max ha investito un milione nell'affare

(2) b. Eva ha investito (molto + tutta se stessa + il suo affetto) nel rapporto con Max

INVESTIRE 3

(3) $\quad \mathrm{N}_{0} \mathrm{VN}_{1}\left(\mathrm{E}+\operatorname{con} \mathrm{N}_{2}\right)=$ :

(3) a. Il generale investí la piazzaforte $(E+$ con le truppe d'assa1to)

(3) b. Luca investí (Eva + I'auto di Eva) (E + con I'automobile)

(3) c. Eva investí Max $(E+(($ con $+d i)$ (tutti $i$ suoi problemi + parolacce))

2. Pour la notion de verbe support, voir Gross (1981). 
INVESTIRE 4

(4) $\quad \mathrm{N}_{0}$ si $\mathrm{V}$ di $\mathrm{N}_{1}=$ :

(4) a. Lotario si investí del potere regale

INVESTIRE 5

(5) $\quad \mathrm{N}_{0}$ si $\mathrm{y}$ di $\mathrm{N}_{1}=$ :

(5) a. Max si è investito del (ruolo + personaggio) di Don Giovanni

INVESTIRE 6

(6) $\quad \mathrm{N}_{0} \mathrm{~V} \mathrm{~N}_{1}=$ :

(6) a. Questa difficile scelta investe tutti noi

(6) b. La trattazione investe anche $i$ pronomi

\section{INVESTIRE 7}

(7) $\quad \mathrm{N}_{0}$ V Prep $\mathrm{N}_{1}=$ :

(7) a. La nave è investita (contro + su) 10 scoglio

\subsection{INVESTIRE 1}

Ce premier emploi se différencie en deux sous-emplois (a et b), ce qui est mis en évidence par l'application du verbe support dare ou de son extension ${ }^{3}$ conferire en cooccurrence avec le $V-n=$ : investitura:

(8) $\quad \mathrm{N}_{0}$ dare Det V-itura di $\mathrm{N}_{2}$ a $\mathrm{N}_{1}$

(8) a. La regina ha (dato + conferito) l'investitura del titolo di baronetti ai Beatles

3. Pour 1a notion de support extension, voir Gross (1981). 
(8) b. *Il governo ha (dato + conferito) l'investitura del compito di indagare sul caso Moro ad una commissione parlamentare

L'inacceptabilité de (8b) est due à des contraintes distributionnelles de $N_{2}$ dans l'exemple (Ib) en cooccurrence avec le $V-n$ investitura. En effet, dans des phrases du type (8a), $N_{2}$ est limité à $N$ du type "titres honorifiques, ecclésiastiques, etc.".

La paraphrase selon (8) permet donc de comfirmer la différence entre les structures (1a) et (1b).

Nous observerons encore qu'à partir de (1), nous pouvons déceler la présence de structures associées avec avere: $\mathrm{N}_{1}$ avere $\mathrm{N}_{2}=$ :

(9) a. I Beatles hanno il titolo di baronetti

(9) b. La commissione parlamentare ha il compito di indagare sul caso Moro

A partir de (8a) nous avons, en outre, avec le Vsup ricevere ${ }^{4}$ :

4. On observera qu'en (9) et (10) le verbe avere apparaît dans deux emplois différents, le premier (9) de "posséder", le second (10) de "recevoir". Ce dernier produit des phrases moins acceptables. Des modifications de temps en (9) et (10) montrent des différences d'ordre aspectuel:

(9) c. I Beatles (ebbero + ricevettero) il titolo di baronetti qui demande une interprétation ponctuelle ou ponctuelle-durative du type

(9) d. I Beatles (ebbero + ricevettero) il titolo di baronetti ne1 1964

avec avere = "recevoir", tandis que

(9) e. I Beatles (ebbero + *ricevettero) il titolo di baronetti soltanto dal 1964 al 1968 , poi ne furono privati ufficialmente a causa di scandali

implique l'emploi de avere = "posséder".

Pour les emplois donner-recevoir, voir Gross (1982). 
(10)

$\mathrm{N}_{1}$ (?avere + ricevere) I'investitura di $\mathrm{N}_{2}$ da $\mathrm{N}_{0}$

(10) a. I Beatles (?hanno + ricevono) 1'investitura del titolo di baronetti dalla Regina

tandis que, pour ( $8 \mathrm{~b}$ ) nous n'avons pas une structure du type (10):

(10) b. *Una commissione parlamentare (ha + riceve) il compito di indagare sul caso Moro dal governo

Nous remarquerons encore que, dans le cas de INVESTIRE 1, les deux emplois (a) et (b) peuvent être paraphrasés par le verbe dare, en modifiant la structure $N_{0} V N_{1} d i N_{2}$ en

$$
\mathrm{N}_{0} \mathrm{~V} \mathrm{~N}_{2} \text { a } \mathrm{N}_{1}=\text { : }
$$

(11) a. La regina diede il titolo di baronetti ai Beatles

(11) b. Il governo diede il compito di indagare sul caso Moro ad una commissione parlamentare

et, seulement pour des phrases du type (b), tout en laissant intacte la structure syntaxique, nous pouvons appliquer une paraphrase avec le verbe incaricare:

$$
\mathrm{N}_{0} \mathrm{VN}_{1} \text { di } \mathrm{N}_{2}=\text { : }
$$

(12) a. *La regina incaricò i Beatles del titolo di baronetti

(12) b. Il governo incaricò una commissione parlamentare del compito di indagare sul caso Moro ${ }^{6}$

Le V-n investitore, dans les structures avec être, est admis

5. Dans ce cas, toute interprétation de avere = "posséder" ou "recevoir" est bloquée, ce qui montre que la structure (12) n'est pas unique et doit être séparée en deux structures.

6. Dans (12b) le syntagme complexe del compito di peut paraître peu naturel, mais il faut considérer une phrase comme

(12) c. Il governo incaricò la commissione parlamentare delle indagini sul caso Moro

qui est plus naturelle. 
d'une façon naturelle pour des paraphrases de (1a) mais non de (1b):

(13) a. La regina è l'investitrice del titolo di baronetti $(\mathrm{E}+$ ?*ai Beatles)

(13) b.?*Il governo è 1 'investitore del compito di indagare sul caso Moro ( $+\star_{a d}$ una commissione parlamentare $)^{7}$

En tout cas, on observera que la conservation de la structure entière, avec $a N_{1}$, est généralement improbable dans des phrases du type (13).

\subsection{INVESTIRE 2}

Cet emploi est différent du précédent, car:

- il n'admet pas la séquence di $\mathrm{H}_{2}$, mais in $\mathrm{N}_{2}$;

- il n'admet pas de construction avec le $V-n$ investitura et avec

le Vsup dare;

- il admet une construction avec le $V-n$ investimento et le Vsup fare, qui n'est pas admise par l'emploi (1).

L'emploi (2) se diffêrencie lui aussi en deux sous-emplois (a, b): $\mathrm{N}_{0}$ fare Det $\mathrm{V}$-imento $\left(\mathrm{di} \mathrm{N}_{1}+\operatorname{Modif}_{1}\right)$ in $\mathrm{N}_{2}=$ :

(14) a. Max ha fatto (un +10 ) investimento di un milione nell'affare

(14) b. Eva ha fatto l'investimento (di tutta se stessa + ?del suo affetto) nel rapporto con Max

(14) c. Eva ha fatto un investimento (?di tutta se stessa + ?del suo affetto + affettivo + narcisistico) nel rapporto con Max

7. Les noms en -tore sont très spécialisés et leur emploi pose souvent des problèmes d'acceptabilité (voir Filoselli, 1983). 
La différence entre (14a) et (14b), en dehors des restrictions sur les déterminants, paraît consister en un écart distributionnel entre deux différentes classes de noms:

a) les "investissements" financiers;

b) les "investissements" psychologiques;

où b) pourrait être interprété comme un emploi métaphorique de a) ${ }^{8}$.

Nous remarquerons que, dans le cas de (14a) comme dans le cas de $(14 \mathrm{~b}, \mathrm{c})$, il est possible d'avoir des structures "passives" avec avere et ricevere et 1 'agent réalisé par da parte di $N_{0}$ :

$\mathrm{N}_{2}$ (avere + ricevere) Det $\mathrm{V}$-imento di $\mathrm{N}_{1}$ da parte
di $\mathrm{N}_{0}=:$

(15) a. L'affare ha (avuto + ricevuto) (un + 1o) investimento di un milione da parte di Max

(15) b. I1 rapporto con Max ha (avuto + ricevuto f'investimento di tutta se stessa da parte di Eva

(15) c. Il rapporto con Max ha (avuto + ricevuto) un investimento affettivo da parte di Eva

\subsection{INVESTIRE 3}

Le troisième emploi est différent des deux premiers, surtout en ce qui concerne les structures (1) et (2) et le $V-n$ investitura, qui ne sont pas admis. Le $V-n$ admis est investimento. Considérons les rêsultats relatifs aux trois sous-emplois discutés:

$$
\mathrm{N}_{0} \text { Vsup Det V-imento di } \mathrm{N}_{1}\left(\mathrm{E}+\operatorname{con} \mathrm{N}_{2}\right)=\text { : }
$$

8. L'emploi (b) est technique et relève du domaine de la psychana1yse, mais il est devenu courant en italien.

9. Ces phrases sont un peu artificielles du fait des enchainements. 
(16) a. Il generale (?*fece + procedette a) I'investimento della piazzaforte $(E+$ con le truppe d'assalto)

(16) b. Luca (*fece + *procedette a) $(10+$ un) investimento di (Max + I'auto di Max) ( $E+\operatorname{con} 1$ a sua auto)

(16) c. Eva (*fece $+*$ procedette a) (1o + un) investimento di Max $(E+\operatorname{con}+d i$ parolacce $)$

Le Vsup fare n'est pas acceptable et le verbe procedere permet une paraphrase acceptable seulement à partir de (3a), c'est-à-dire avec investire = "assiéger", "attaquer", et non à partir de (3b) et (3c), pour lesquels on obserbe un cas d'anomalie sémantique (notée *), c'est-à-dire une interprétation presque "humoristique".

On observera que l'emploi (3a) est le plus ancien, désormais uniquement technique, tandis que (3b) est l'emploi le plus ordinaire ("collision" entre voitures ou "1e fait de renverser un piéton"), et (3c) est un emploi métaphorique.

À partir de $(3 \mathrm{a}, \mathrm{b}, \mathrm{c})$ nous pouvons constater une relation systématique du type

$$
\mathrm{N}_{0} \mathrm{~V} \mathrm{~N} \mathrm{~N}_{1} \operatorname{con} \mathrm{N}_{2}=\mathrm{N}_{2} \mathrm{~V} \mathrm{~N}_{1}=:
$$

(17) $a_{.}=$Le truppe d'assalto investirono la piazzaforte

(17) $b_{.}=$La sua auto investí (Eva + 1'auto di Max)

(17) $c_{.}=$Parolacce investirono Max 10

10. (17c) est plus naturel au passif:

(17) d. Eva fu investita da parolacce On observe que la structure (c) permet un emploi avec la préposition $d i$ au lieu de con:

(17) e. Eva investì Max di parolacce ainsi qu'au passif:

(17) f. Max fu investito di parolacce (E + da Eva)

Les autres structures ne permettent pas cet emploi: 
En revenant à investimento, à partír de $(3 \mathrm{~b})$ et non de $(3 c)$, nous avons une structure avec Vsup =: fare sans $N_{1}$ :

(18) $\quad \mathrm{N}_{0}$ fare un $\mathrm{V}$-imento $\operatorname{con} \mathrm{N}_{2}=$ :

(18) b. Luca ha fatto un investimento con la sua auto

et une structure "passive" avec Vsup =: subire sans con $N_{2}$ et sans $N_{0}:$

(19) $\mathrm{N}_{1}$ subire un $\mathrm{V}$-imento $=$ :

(19) b. (Eva + I'auto di Eva) ha subito un Investimento

C'est surtout 1'emploi ( $3 \mathrm{~b}$ ) qui admet une paraphrase avec le $\mathrm{V}-n$ investitore et le $V-n$ investito :

(20) $\mathrm{N}_{0}$ essere Det V-itore di $\mathrm{N}_{1}=:$
(20) b. Luca è 1 'investitore di (Eva + 1'auto di Eva)

et

(21) $\mathrm{N}_{1}$ essere Det V-ito =:

(21) b. Max è 1'investito

Avec des degrés d'acceptabilité variés, nous pouvons étendre les structures (20) et (21) aux emplois (3a) et (3c).

Comme les aurres, ces trois sous-emplois sélectionnent des classes de $N$ différentes.

\subsection{INVESTIRE 4}

Cet emploi pourralt être analysé à partir de (1), par une réflexivation telle que $N_{1}=N_{0}=s i$ :

(17) g. *I1 generale investi la piazzaforte di truppe d'assalto

(17) h. *Eva invest I Luca della sua auto 


$$
\mathrm{N}_{0} \mathrm{~V} \mathrm{~N}_{1} \text { di } \mathrm{N}_{2}=:
$$

(22) a. *Luca investí Luca del potere regale

(22) b. réflex = Luca si investí del potere regale

dans le sens de Luca si auto-investi del potere regale

L'emploi (1) n'admet pas la structure de type (22b) car celle-ci n'a qu'une interprétation comme

$$
\text { Luca (si approprid di + usurpo) il potere regale }
$$

impossible pour (1). Pour ces raisons (sêmantiques et de coréférence), i1 est peut-être nécessaire de considérer cette entrée comme INVESTIRSI plutôt que comme INVESTIRE 4 .

Toutes les nominalisations sont interdites ict.

\subsection{INVESTIRE 5}

Cet emploi se diffêrencie du prêcédent seulement par 1a présence d'une séquence lexicalement constante, c'est-à-dire quasi-figẻe, composée par Det molo di $N$ et Det personaggio $d i N$ à $l$ 'intérieur du groupe $N_{1}$ :

$$
\begin{aligned}
& \mathrm{N}_{0} \text { si } \mathrm{V} \text { di (Det (ruolo + personaggio) di } \mathrm{N}_{1} \text { ) }=: \\
& \text { a. *(Luca }+11 \text { teatro) investe Max del (ruolo + personag- } \\
& \text { gio) di Don Giovanni }
\end{aligned}
$$

On remarquera que dans ce cas aussi la coréférence $N_{0}=N_{1}$ est ob1igatoire et donc la nếcessitể de considérer une entrée INVESTIRSI 2 plutôt qu'une entrée INVESTIRE 5 .

Les nominalisation sont interdites ici également. 


\subsection{INVESTIRE 6}

Cet emploi n'admet, à la différence de (1) et (2), ni complément di $N_{2}$, ni Loc $N_{2}$, ni structures avec Vsup et $V-n$. Ce qui le caractêrise est la classe des $N$ en position $N_{0}$, qui doivent être $N n r$, c'està-dire "non actifs"11:

$$
(\mathrm{Nnr}+\mathrm{Ch} \mathrm{F}+\mathrm{V} \text {-inf })_{0} \mathrm{VN}_{1}=\text { : }
$$

(26) a. (La scelta elettorale + Il fatto che si debba votare + Salvare una vita) investe (Max + tutti noi)

dans ce cas $N_{1}=:$ Hum, mais 1 'emploi (6b) implique la possibilité de $N_{1}=: \quad N-2 m:$

(26) b. (La trattazione + I1 fatto che ci sia accordo + La coreferenza) investe $i$ pronomi

Les nominalisatins sont interdites une fois de plus.

\subsection{INVESTIRE 7}

Cet emploi technique appartenant au langage de la mer concerne le fait qu'une embarcation puisse s'échouer ou heurter le fond de la mer ou des rochers. L'auxiliaire essere confirme un emploi "intransitif" ou prépositionne1. Le $V-n$ investimento est attesté dans des phrases comme

$\mathrm{N}_{0}$ Vsup V-imento $\left(E+\right.$ Prép $\left.\mathrm{N}_{1}\right)=$ :

(27) a. La nave fece imvestimento (E + sullo scoglio)

11. Pour les notions de "actif", "non actif", "non restreint", "humain" et "non humain", voir Gross (1975). 
3. Les emplois verbaux et nominaux du morphème INVEST- en français Nous pensons que la traduction en français des exemples italiens étudiés fournit un autre type d'argumentation en faveur des séparations que nous avons faites. De plus, cette traduction pourraît être vue comme une première tentative de comparaison entre les structures italiennes et celles du français. Nous mettons donc en correspondance les emplois français avec les emplois italiens, tout en signalant qu'il s'agit là d'un même morphème avec des réalisations différentes dans les deux langues. Nous examinerons les emplois français selon 1 'ordre de présentation des emplois italiens:

INVESTIR 1

$$
\mathrm{N}_{0} \vee \mathrm{N}_{1} \text { de } \mathrm{N}_{2} \text { : }
$$

(28) a. Le pape a investi l'évêque du titre cardinalice

(28) b. Le gouvernement a investi le ministre d'une charge importante

INVESTIR 2 $\mathrm{N}_{0} \mathrm{~V} \mathrm{~N}_{1}$ dans $\mathrm{N}_{2}$ :

(29) a. Max a investi un million dans 1'affaire

(29) b. Éva a Investi (beaucoup + son affection) dans le rapport avec Max

\section{INVESTIR 3}

$$
\mathrm{N}_{0} \vee \mathrm{N}_{1}\left(\mathrm{E}+\operatorname{avec} \mathrm{N}_{2}\right) \text { : }
$$

(30) a. Le général a investi la ville ( + (avec ses troupes)) 


\subsection{INVESTIR 1}

Ce premier emplo1 se différencie, comme pour l'italien, en deux sous-emplois ( $a$ et $b$ ) et, si $1^{\prime}$ on construit une paraphrase avec le verbe support donner et le $V-n$ investiture on a presque les mêmes résultats que pour $1^{\prime}$ italien:

$$
\mathrm{N}_{0} \text { Vsup V-iture de } \mathrm{N}_{2} \text { à } \mathrm{N}_{1} \text { : }
$$

(3I) a. Le pape a donné 1'investiture du titre cardinalice à l'évêque (Petit Robert)

(31) b. *Le gouvernement a donné 1 'investiture d'une charge importante au ministre

En français, néamnoins, il y a deux emplois techniques avec donner et investiture qui ne dépendent pas de la structure (28a):

$$
\text { c. }\left\{\begin{array}{l}
\text { Le parti a investi son candidat } \\
\text { Le parti a donné } 1 \text { 'investiture à son candidat }
\end{array}\right.
$$

(28) d. $\left\{{ }^{*}\right.$ 'assemblée a investi le ministre L'assemblée a donné I'investiture au ministre

En outre, à partir de (28b), on observe une possibilité de cooccurrence d'entrées lexicales en position $N_{2}$ plus large qu'en italien, étant donné qu'on peut avoir des phrases comme

(28) e. Max a investi Luc de toute sa confiance sans possibilité de constructions avec Vsup:

(31) c. *Max a donné l'investiture de sa confiance à Luc Mais (28e) n'est pas acceptable en italien: *Max ha investito Luc di tutta la sua fiducia

alors qu'en revanche on a: 


\subsection{INVESTIR 2}

Les deux emplois français ne se différencient pas en deux sousemplois par les constructions avec Vsup faire et V-n investissement; il n'est, en effet, possible d'avoir une paraphrase avec verbe support que pour 1'emploi (a): $\mathrm{N}_{0}$ Vsup V-issement de $\mathrm{N}_{1}$ dans $\mathrm{N}_{2}=$ :

(34) a. Max a fait (un $+1^{\prime}$ ) investissement d'un million dans 1'affaire

(34) b. *Ẻva a fait (un $+1^{\prime}$ ) investissement de son affection dans le rapport avec Max

On peut remarquer que le nom investissement est employé seulement à un niveau strictement technique dans le domaine psychanalytique.

Pour l'emploi (a), il y a aussi une construction avec Vsup être et $V-n$ investisseur:

$$
\mathrm{N}_{0} \mathrm{~V} \text {-sup V-isseur de } \mathrm{N}_{1} \text { dans } \mathrm{N}_{2}=\text { : }
$$

(35) a. Max est 1'investisseur (?d'un million) dans l'affaire

\subsection{INVESTIR 3}

L'emploi (3) en français est plus restreint qu'en italien, étant donné qu'on a seulement une classe de $N_{0}=$ : Nhumain, militaire et que pour les emplois (b et $c$ ) de 1'italien, on a des correspondants différents :

(36) b. Luc a renversé le piéton avec sa voiture

(37) b. Luc a heurté la voiture de Paul

(38) b. Le camion de Luc est entré en collision avec celui de Paul 
(39) c. Éva a courvert Max de (insultes, gros mots)

On n'enregistre aucun emploi avec Vsup faire ou procéder et $V-n$ investissement, $\mathrm{ni}$ avec Vsup être et $V-n$ investisseur.

3.4 Les correspondants en français des autres emplois d'investire italien

Les autres emplois verbaux d'investire, y compris les emplois mentionnés dans le paragraphe précédent, ont les correspondants suivants:

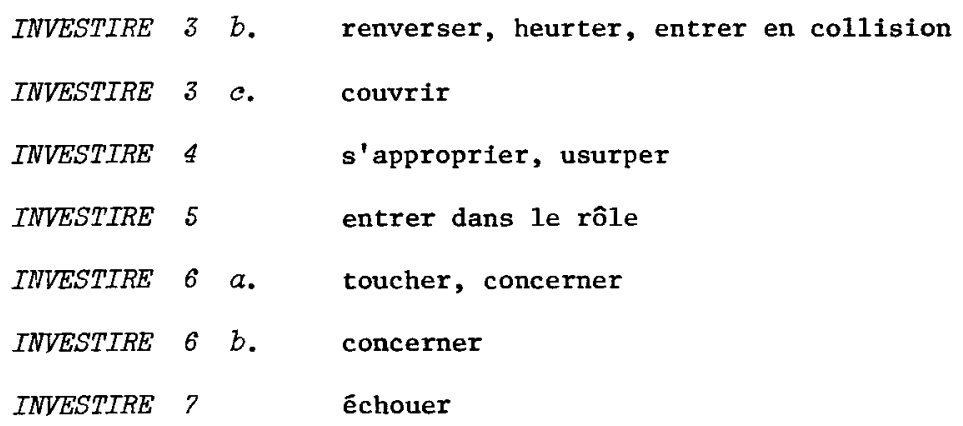

4. Esquisse de représentation comparative des données italiennes et françaises

Nous avons élaboré deux tableaux comparatifs des réalisations du morphème INVEST- en italien et en français. Le premier (Tableau 1) permet d'avoir une vue d'ensemble des correspondances. Le deuxième tableau permet une comparaison détaillée des propriétés syntaxiques distributionnelles et des paraphrases avec Voup et $V-n$. 

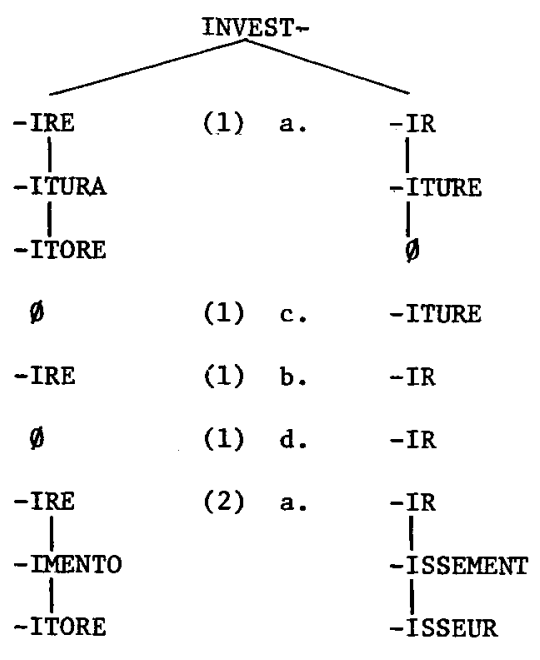

-IRE (2) b. -IR

$\left.\right|_{- \text {ITORE }} ^{\text {-IRE }}$

(3) a<smiles>O=[Ru]O</smiles>

-IRE (3) b.

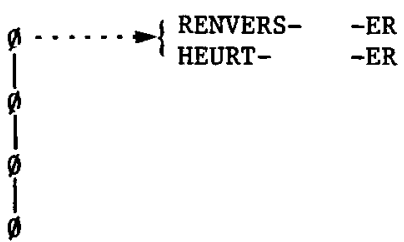

-IRE

(3) c.

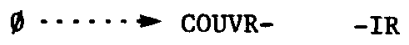

-IRE

(4)

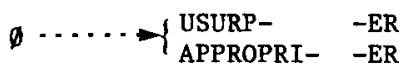

-IRE

(5)

$\emptyset$

ENTR-

-ER

-IRE

(6) $a$.

$\emptyset \cdots \cdots \rightarrow \begin{cases}\text { TOUCH- } & \text {-ER } \\ \text { CONCERN- } & \text {-ER }\end{cases}$

-IRE

(6) b,

$\emptyset . .$. CONCERN- -ER

$-\mathrm{TRE}$

(7)

$\emptyset . . .$. ÉCHOU- -ER

-TMENTO 


\section{Conclusions et perspectives}

\subsection{L'unité du mot}

L'exemple d'analyse des structures de phrase simple que nous avons donné montre que la notion de mot et surtout celle d'unité du mot n'a qu'une valeur phonologique. En effet, au niveau morpho-phonologique comme au niveau syntaxique, on a pu observer que 1 e(s) mot(s) investire ou le(s) mot(s) investir n'ont pas seulement des sens différents, mais qu'ils entrent dans des structures syntaxiques différentes; ils admettent des classes distributionnelles de $N$ différentes et ont des relations de morphologie dérivationnelle différentes.

Nous avons montré, en outre, qu'on peut éliminer ${ }^{12}$ de la procédure le recours au sens, étant donné que pour chaque interprétation sémantique différente nous avons étayé chaque emploi a'au moins une propriété syntaxique spécifique. En conséquence, les verbes, en tant qu'entrées du lexique, doivent être considérês comme des "phrases simples", car chaque mot est utilisé dans un nombre limité de combinaisons syntaxiques. Si cela est vrai pour les verbes (et pour les adjectifs), nous avons montré que, pour les noms, l'étude des structures de phrase à verbe support permettait de déceler un nombre important de propriétés syntaxiques que les analyses classiques n'ont jamais pu mettre en évidence (au moins à un niveau systêmatique et exhaustif).

12. L'utilisation heuristique des différences de sens est utile, mais les sens n'ont pas de représentation formelle, dans l'état actuel de la théorie des lexiques-grammaires. 


\subsection{Problemes de comparaison}

Le Lexique-Grammaire d'une langue fournit des informations systématiques comme celles que nous avons présentées dans cet essai. I1 s'agit, pour les entrées du lexique, de propriétés structurelles et transformationnelles ainsi que distributionnelles et paraphrastiques. Cela implique que $1^{\prime}$ on dispose aussi d'informations systématiques sur les conditions d'application des "règles" syntaxiques, notamment des conditions "lexicales".

La tradition linguistique dans le domaine de la comparaison est assez pauvre en regard des aspects syntaxiques. En effet, on peut dire qu'à part les ouvrages lexicographiques bilingues, axés sur 1a sémantique et qui contiennent seulement des indications implicites d'ordre syntaxique, jusqu'à maintenant la séparation académique et scientifique des domaines du lexique et de la syntaxe n'a pas favorisé les études de syntaxe comparée. La constitution de plusieurs LexiquesGrammaires permet aux chercheurs d'ouvrir un paradigme important dans les sciences linguistiques: la syntaxe comparée basée sur une connaissance approfondie du lexique, et donc des conditions lexicales sur 1 'application des "règles". Les travaux des équipes françaises, italiennes, espagnoles, portugaises ont permis de commencer des recherches de syntaxe comparée des langues romanes ${ }^{13}$.

Parmi d'autres stratégies de recherches, il y en a au moins deux

13. Deux colloques ont dêjà eu lieu (1981, à Palermo; 1982, à La Croix en Touraine) qui ont permis aux chercheurs des différentes équipes travaillant sur les lexiques-grammaires de commencer à expérimenter des techniques de comparaison syntaxique. Voir Lamiroy (1981, 1983). 
qui se prēsentent comme très stimulantes:

a) I'êlaboration d'hypothèses globales concernant des domaines compacts, soit au niveau structural, soit au niveau lexical ${ }^{14}$;

b) la comparaison lexico-syntaxique détaillée axée sur 1'étude des correspondances entre structures syntaxiques et classes d'entrées lexicales.

Ce que nous avons présenté est une comparaison détaillée sur un exemple transparent de correspondance êtymologique morphologique. En effet, il n'y a qu'un noyau limité qui se prêsente de cette façon: cependant, même ce noyau reste inconnu dans les détails et surtout en relation avec des propriétés syntaxiques analysées avec une méthodologie commune.

Il faut souligner que les données de chaque Lexique-Grammaire sont mémorisées dans des banques de données informatiques reliées entre e1$1 e s^{15}$. Cela permettra un accès rapide aux données et selon différents points d'observation: ce qui signifie, par exemple, qu'on peut baser une recherche sur les entrées aussi bien que sur les propriétés, chacune prise singulièrement ou regroupées selon 1 'intention du chercheur. Le temps est venu de voir clairement, et de très près, ce quil se passe dans le lexique et la grammaire de langues différentes. L'étude des langues romanes, nous 1'espérons bien, ouvrira le chemin pour d'autres recherches plus complexes encore, telles que la comparaison des lan-

14. Voir par exemple, Elia (1983).

15. Ces banques de données exploitent le système LEXSYN du L.A.D.I., voir Vasseux (1983). 
gues indoeuropéennes ou des langues du monde.

Annibale Elia

Istituto di Linguistica dell' Universita

di Salerno

Centro di Informatica Linguistica 


\section{RËFÉRENCES}

ELIA, A., M. MARTINELLI et E. D'AGOSTINO (1981) (EMDA) Lessico e strutture sintattiche, Napoli Liguori.

ELIA, A. (1983) "Une hypothèse globale sur les verbes à compléments locatifs et sur l'infinitive locative", in Lingua e stile (à paraftre).

FILOSELLI, E. (1983) I nomi in -tore, tesi di Laurea, Salerno.

GROSS, G. (1982) "Un cas de construction inverse: "Donner" et "Revevoir", Linguisticae Investigationes, vol. 6, no 1 .

GROSS, M. (1975) Méthodes en syntaxe, Paris, Hermann.

GROSS, M. (1981) "Les bases empiriques du prédicat sémantique", in Langages, vo1. 63.

LAMIROY, B. (1981) Les verbes de mouvement suivis de l'infinitif en français, en espagnol et en néerzandais, thèse de doctorat, Katholieke Universiteit Leuven.

LAMIROY, B. (1983) La Zinguistique comparée et l'argronentation en syntaxe, in $\mathrm{C}$. Angelet et ai. (eds.), Langue, dialecte, littératu$r e$, Leuven University Press.

VASSEUX, P. (1983) Systeme LEXSYN, L.A.D.L., Paris. 
TABLEAU 2

\begin{tabular}{|c|c|c|c|c|c|c|c|c|}
\hline 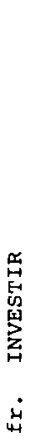 & 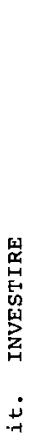 & & & 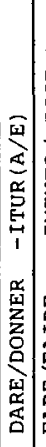 & 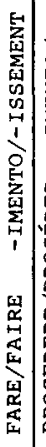 & 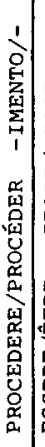 & 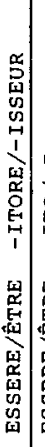 & 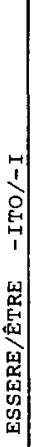 \\
\hline+ & + & $\mathrm{N}_{0} \mathrm{VN}_{1}(\mathrm{di} / \mathrm{de}) \mathrm{N}_{2}=;$ titre & (a) & & & & & \\
\hline+ & + & $\mathrm{N}_{0}$ Vsup $\mathrm{V}-\mathrm{n}(\mathrm{di} / \mathrm{de}) \mathrm{N}_{2} \mathrm{~A} \mathrm{~N}_{1}$ & & + & & & + & \\
\hline+ & + & $\mathrm{N}_{0} \mathrm{~V} \mathrm{~N}_{1}(\mathrm{di} / \mathrm{de}) \mathrm{N}_{2}=\mathrm{i}$ charge & (b) & & & & & \\
\hline- & - & $\mathrm{N}_{0}$ Vsup $V-\mathrm{n}(\mathrm{di} / \mathrm{de}) \mathrm{N}_{2} \mathrm{~A} \mathrm{~N}_{1}$ & & & & & & \\
\hline- & - & $\mathrm{N}_{0} \mathrm{~V} \mathrm{~N} \mathrm{~N}_{1}$ & (c) & & & & & \\
\hline+ & - & $\mathrm{N}_{0}$ Vsup V-n A $\mathrm{N}_{1}$ & & + & & & & \\
\hline+ & - & $\mathrm{N}_{0} \mathrm{~V} \mathrm{~N} \mathrm{~N}_{1}(\mathrm{di} / \mathrm{de}) \mathrm{N}_{2}^{0}=:$ confiance & (d) & & & & & \\
\hline - & - & $\mathrm{N}_{0}$ Vsup $\mathrm{V}-\mathrm{n}(\mathrm{di} / \mathrm{de}) \mathrm{N}_{2} \mathrm{~A} \mathrm{~N}_{1}$ & & & & & & \\
\hline+ & + & $\mathrm{N}_{0} \mathrm{~V} \mathrm{~N} \mathrm{~N}_{1}=: \quad \$($ in/dans $) \mathrm{N}_{2}$ & (a) & & & & & \\
\hline+ & + & $\mathrm{N}_{0}$ Vsup $V-n(d i / d e) N_{1}$ (in/dans) $N_{2}$ & & & + & & + & \\
\hline+ & + & $\mathrm{N}_{0} \vee \mathrm{N}_{1}=: \quad \psi($ in/dans $) \mathrm{N}_{2}$ & (b) & & & & & \\
\hline- & + & $\mathrm{N}_{0} V \sup V-\mathrm{n}(\mathrm{di} / \mathrm{de}) \mathrm{N}_{1}(\mathrm{in} /$ dans $) \mathrm{N}_{2}$ & & & + & & & \\
\hline+ & + & $\mathrm{N}_{0}=:$ militaire $\mathrm{V} \mathrm{N} \mathrm{N}_{1}\left(\mathrm{E}+\left((\right.\right.$ con/avec $\left.\left.) \mathrm{N}_{2}\right)\right)$ & (a) & & & & & \\
\hline- & + & $\mathrm{N}_{0}$ Vsup V-n (di/de) $\mathrm{N}_{1}$ Comp & & & & + & & \\
\hline- & + & $\mathrm{N}_{0} \mathrm{VN}_{1}\left(\mathrm{E}+\left((\right.\right.$ con $/$ avec $) \mathrm{N}_{2}^{0}=:$ voiture $\left.)\right)$ & (b) & & & & & \\
\hline- & + & $\mathrm{N}_{0} V \sup V-n\left(E+\left((\right.\right.$ con $/$ avec $\left.\left.) \mathrm{N}_{2}\right)\right)$ & & & + & & & \\
\hline- & + & $\mathrm{N}_{1}$ Vsup $\mathrm{V}-\mathrm{n}$ & & & & & + & + \\
\hline- & + & $\mathrm{N}_{0} \mathrm{~V} \mathrm{~N} \mathrm{~N}_{1}\left(\mathrm{E}+\left((\right.\right.$ con/avec $) \mathrm{N}_{2}=:$ métaph. $\left.)\right)$ & (c) & & & & & \\
\hline- & - & $\mathrm{N}_{0}$ Vsup V-n $(\mathrm{di} / \mathrm{de}) \mathrm{N}_{1}$ Comp & & & & & & \\
\hline
\end{tabular}


TABLEAU 2*

\begin{tabular}{|c|c|c|c|c|c|c|c|c|}
\hline 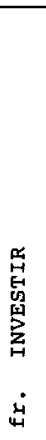 & 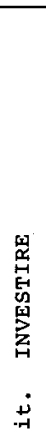 & & & 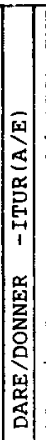 & 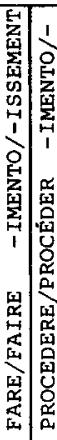 & 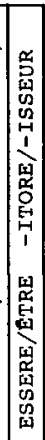 & 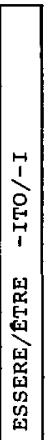 & \\
\hline- & + & $\mathrm{N}_{0} \mathrm{si} \mathrm{V}$ di $\mathrm{N}_{1}$ & & & & & & 4 \\
\hline- & + & $N_{0} s i$ V di Dét $C_{1}(d i / d e) N_{2}$ & & & & & & \\
\hline- & + & $\mathrm{c}_{1}=:$ ruolo, personaggio/rôle, $\ldots$ & & & & & & o \\
\hline- & + & $\mathrm{Nnr}_{0} \mathrm{~V}$ Nhum $_{1}$ & (a) & & & & & \\
\hline- & - & $\mathrm{N}_{0}$ Vsup $\mathrm{V}-\mathrm{n}(\mathrm{di} / \mathrm{de}) \mathrm{N}_{1}$ & & & & & & 8 \\
\hline- & + & $\mathrm{Nnr}_{0} \mathrm{~V}$ N-hum ${ }_{1}$ & (b) & & & & & 0 \\
\hline- & - & $\mathrm{N}_{0}$ Vsup $\mathrm{v}-\mathrm{n}(\mathrm{di} / \mathrm{de}) \mathrm{N}_{1}$ & & & & & & \\
\hline- & + & $\mathrm{N}_{0} \vee\left(E\left(\right.\right.$ Prép $\left.\left.\mathrm{N}_{1}\right)\right)$ & & & & & & 7 \\
\hline- & + & $\mathrm{N}_{0}$ Vsup V-n Comp & & & + & & & 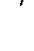 \\
\hline
\end{tabular}

* Ce tableau doit lu à partir de la gauche en haut où sont situés les verbes investire et investir. Au centre les propriétés sont alignées et marquées par des indications situées à droite concernant le numéro et le type d'emploi (exemple, (a) ... 1, (b) ... 3, etc.). En haut à droite, il y a des spécifications sur les $V$ sup et les $V$ - $n$.

Les structures équivalentes, italien-français, pour les prépositions et les verbes supports, comportent une représentation avec barre / (exemple, di/de: di pour l'italien et de pour le français; dare/donner: dare pour l'italien et donner pour le français). À gauche, il y a deux colonnes avec des plus et des moins $(+,-)$ qui indiquent si la propriété en question est valable ou non pour le verbe; s'il s'agit d'une propriété avec $V s u p$, il faut d'abord voir si le verbe à gauche a un + ou un - dans la case de la colonne; si c'est + alors on peut lire à droite en haut le type de $V$ sup et de $V-n$; si c'est -, mais s'il y a un + à droite, cela veut dire qu'il se réfere seulement à un des deux verbes.

Le symbole $\$$ est équivalent à "investissements financiers" et le symbole $\Psi$ à "investissements psychologiques". 